\section{Application of scanning electron microscopy to the study of skin window preparations}

R. J. SOKOL, T. E. DURRANT, AND G. HUDSON University Department of Haematology, Royal Infirmary, Sheffield S6 3DA

Skin window preparations have been increasingly used to study the cellular inflammatory response in a variety of haematological conditions (Ghosh et al, 1973) but little attention seems to have been given to the surface features of cells in this situation. This report describes a method which has been developed for studying skin window cells with the scanning electron microscope (SEM).

\section{Material and methods}

Skin window preparations are made on the anterior aspect of the forearm, using a method already described in detail (Ghosh et al, 1973). After careful cleaning of the skin with soap and spirit, the epidermis is scraped away over a small area with a sterile scalpel blade until exudation is noted. Loose squames are wiped away and a sterile coverslip is applied. For this purpose a circular coverslip of 13 $\mathrm{mm}$ diameter is used. A cardboard square is placed over the coverslip for protection and secured with surgical tape. Serial examination of preparations can be carried out by removing coverslips at appropriate intervals and replacing them with fresh ones. Immediately after removal the coverslip is washed in sterile isotonic saline in a Petri dish for about 10 minutes. The specimen is then fixed by transferring the coverslip to another Petri dish containing fixative.

The fixative used is $1.5 \%$ glutaraldehyde in phosphate buffer $\mathrm{pH} 7 \cdot 3$ freshly made from stock solutions A and B. Solution A consists of $15.6 \mathrm{~g}$ per litre of $\mathrm{NaH}_{2} \mathrm{PO} 4.2 \mathrm{H}_{2} \mathrm{O}$, and solution B of $35.8 \mathrm{~g}$ per litre of $\mathrm{Na}_{2} \mathrm{HPO} 4.12 \mathrm{H}_{2} \mathrm{O}$, the solutions being mixed in a proportion of $\mathrm{A}: \mathrm{B}, 23: 77$.

After fixation for 45 minutes the coverslip is transferred to another Petri dish containing sterile, particle-free distilled water and washed for about $10-15$ minutes. It is then slowly dried by being left in a wet chamber overnight.

Handling of the coverslip in the above procedures is facilitated by placing it immediately after removal in a small metal cap with holes punched in it.
After drying, the coverslip is mounted on an SEM stub with high conductivity paint (DAG 915) and the specimen is coated with $60 / 40$ gold/palladium in a vacuum coating unit on an orbital jig (Balzer micro BA3).

The specimen is examined in a scanning electron microscope (Cambridge $\mathrm{S} 4$ at $10 \mathrm{kV}$ using a viewing angle of $45^{\circ}$ ).

Parallel preparations can be obtained from a second abrasion on the same forearm and examined by light microscopy, using the method already described (Ghosh et al, 1973).

\section{Results and discussion}

With the method described consistent appearances are obtained. The figure illustrates the features of macrophages at 48 hours. To the best of our knowledge, SEM techniques have not previously been used in published studies of the cellular inflammatory reaction in skin windows. The particular advantage of the technique is that it enables the surface of cells to be examined in what is effectively an in vivo environment.

Certain technical points appear important. To avoid artefact it has been found essential to prevent the specimen drying prior to fixation. Washing of the specimen with saline at this stage prevents drying and gives a cleaner background. Following fixation and washing, slow drying in a wet chamber

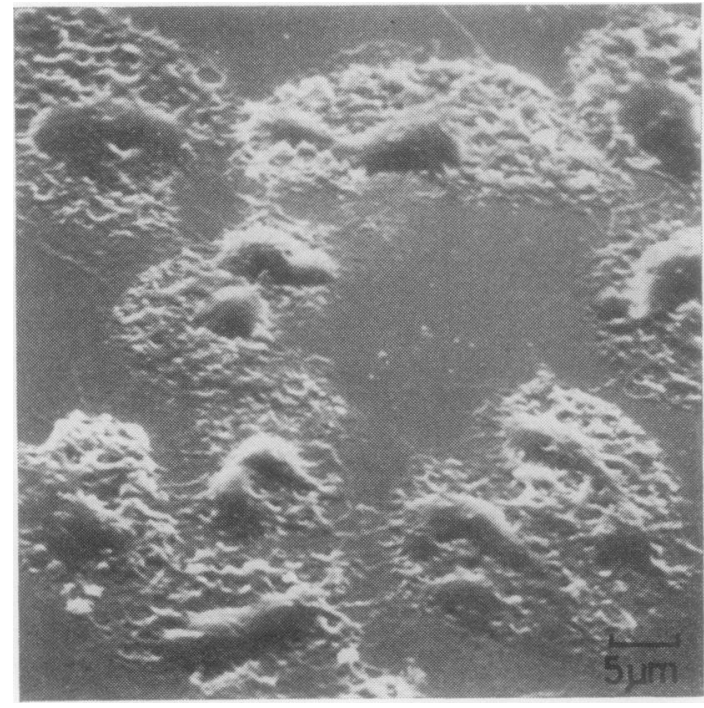

Figure SEM appearances of a skin window preparation at 48 hours, showing a group of macrophages. Features of nuclei, cytoplasm, and surface outline are apparent. 
has been found a satisfactory way of preserving the quality of the preparations, giving superior results to simple air drying which causes distortion and shrinkage of cells with considerable loss of surface detail. Critical point drying which has also been shown to be superior to simple air drying in this respect (Polliack et al, 1973) can be used with the present method, but special facilities are required.

Preliminary observations using the method indicate its potential value in studying cellular function in the inflammatory reaction in patients with malignant blood disorders and other disease states.
This work was supported by the Yorkshire Council for Cancer Research.

\section{References}

Ghosh, M. L., Hudson, G., and Blackburn, E. K. (1973). Skin window macrophages in malignant lymphomas. Brit. J. Haemat., 25, 293-297.

Polliack, A., Lampon, N., and De Harven, E. (1973). Comparison of air drying and critical point drying procedures for the study of human blood cells by scanning electron microscopy. Proceedings of the 6th Annual Scanning Electron Microscopy Symposium, Illinois Institute of Technology, Chicago, pp. 529-534.

\section{Letters to the Editor}

Rheovirus and E. coli in infantile enteritis The recent article by Bishop et al (1976) reports inconclusive results which may be interpreted in different ways. These authors studied an outbreak of acute enteritis in the neonatal wards of a Melbourne hospital; 32 babies were at risk and 15 developed diarrhoea. Escherichia coli $\mathrm{O} 111$. H21 was found in 11 babies with diarrhoea and in five of those without diarrhoea, while a reovirus-like agent was found in eight babies with diarrhoea and in three without diarrhoea. On the basis of these results the authors conclude "that the epidemic was primarily caused by infection with the reovirus-like agent' and they 'do not consider that the strain of $E$. coli 0111 isolated in this study was primarily implicated in the aetiology of the epidemic'. E. coli O111 was isolated three days before the onset of diarrhoea in two babies, coincident with onset in four babies, and seven days after onset in one baby. In four cases the relation of acquisition to onset of symptoms was not determined. On the basis of these observations it was concluded that 'Isolation from rectal swabs (of $E$. coli O111) did not correlate with the presence and absence of symptomatic illness'. No attempt was made to relate the acquisition of reoviruslike particles to the onset of symptoms. The authors seem willing to apply the principles of epidemiology only in support of the pathogenicity of reovirus. However, the same principles should be applied to enteropathogenic $E$. coli, and the evidence from this outbreak could suggest equally well that $E$. coli 0111 was an epidemic agent.

Dr Bishop and her colleagues failed to demonstrate enterotoxin production by the strains of $E$. coli $\mathrm{O} 111 . \mathrm{H} 21$ and use this finding in support of their conclusion. However, enterotoxin production should not be considered as the sole criterion of enteropathogenicity. We have studied epidemic strains of $E$. coli from welldocumented, notorious outbreaks of infantile enteritis in the United Kingdom and none of these strains could be shown to produce enterotoxin using the infant mouse and the $\mathrm{Y} 1$ and $\mathrm{CHO}$ tissue culture systems (Gross et al, 1976). Nevertheless there is good epidemiological evidence to support the aetiological role of these epidemic strains in the outbreaks. We consider that this epidemiological evidence establishes the enteropathogenicity of the strains which we studied, and the failure to demonstrate enterotoxigenicity does not invalidate this conclusion. These strains may produce enterotoxin at a level below that detectable by the current test systems or may produce an enterotoxin which differs qualitatively from the enterotoxin detected by these tests. Alternatively, enterotoxin may not play a part in the pathogenesis of infantile enteritis caused by enteropathogenic $E$. coli.

It is an admirable aim to search for new causes of disease but it is a dangerous policy to minimize the importance of established pathogens. Bacteriologist must remain alert to the danger of out breaks of infantile enteritis due to $E$. cole those involved in the Teesside (Lance? 1968; British Medical Journal, 1968) Manchester (Jacobs, 1969; Jacobs et af 1970), and Glasgow (Rowe and Gross 1971; Love et al, 1972; Kennedy et als 1973) outbreaks are well aware of the virulence of some strains of $E$. coli.

B. ROWE, R. J. GROSS, angी SYLVIA M. SCOTLAND Salmonella and Shigella Reference Laboratory Central Public Health Laboratory Colindale Avenue London NW9 5HT

\section{References}

Bishop, R. F., Hewstone, A. S., Davidson G. P., Townley, R. R. W., Holmes, I. Ho and Ruck, B. J. (1976). An epidemic of diarrhoea in human neonates involving â reovirus-like agent and "enteropathogenices serotypes of Escherichia coli.J. clin. Path 29, 46-49.

British Medical Journal (1968). Minister statement on Tees-side gastroenteritise Brit. med. J., 1, 715.

Gross, R. J., Scotland, S. M., and Rowe. BD (1976). Enterotoxin testing of Escherichid coli causing epidemic infantile enteritis if the U.K. Lancet, 1, 629-631.

Jacobs, S. I. (1969). In Report on Gastro® enteritis, edited by F. N. Marshall, p. 255 Department of Health and Social Security London. 